\title{
NUEVOS CULTIVARES DE MAÍz CON POTENCIAL AGRONÓMICO PARA EL TRÓPICO HÚMEDO 1
}

\author{
Mariano Mendoza², Arnoldo Oyervides ${ }^{2}$, Alfonso López ${ }^{2}$
}

\begin{abstract}
RESUMEN
Nuevos cultivares de maíz con potencial agronómico para el trópico húmedo. Los objetivos de este estudio fueron evaluar el comportamiento de un grupo de líneas considerando su aptitud combinatoria, determinar los mejores híbridos triples y medir la heterosis útil que existe en las características bajo estudio. Para ello se realizó la evaluación de 23 líneas con dos probadores; el experimento se estableció en dos repeticiones y dos localidades en Veracruz, México durante 1995 y se utilizó un diseño bloques al azar con arreglo factorial, tomando como factor A las líneas y factor B los probadores. La respuesta promedio de líneas, probadores y línea x probador resultó estadísticamente diferente para las variables días a floración, altura de planta, prolificidad y rendimiento. En promedio sobresalieron la línea 37 y siete con 9.473 y 9.203 t/ha, respectivamente. Los mejores híbridos mostraron rendimientos entre 9.173 a $9.685 \mathrm{t} / \mathrm{ha}$ y participan las líneas 7, 15, 17, 37, 39 y 49. La heterosis más alta y la mejor ACE se encontró en la cruza de la línea 17 x probador 1 con 18,34 \% para rendimiento; para esta misma variable la mejor ACG $(0,52)$ fue para la línea 37 . La heterosis para floración fue de $-7,27 \%$ y sobresalieron por su ACG las líneas 14, 15 y 39. Para prolificidad la línea 31 presentó el valor más alto de heterosis (52\%); este mismo material mostró la mejor ACG; y para ACE la línea 17 x probador 1 fue la más sobresaliente $(6,28)$.
\end{abstract}

\begin{abstract}
New maize cultivars with agronomic potential for the humid-tropics. It was studied the performance of each of group of lines based on their combining ability to determine the best hybrids. We also estimated usefull heterosis in the traits under study. 23 lines were crossed with two testers and evaluated in 1995 in a experiment with two replications in two locations in Veracruz, Mexico. A complete block design with factorial arrangement was utilized. Lines were considered as factor A while testers were considered as factor B. Results indicated that the average performance of lines, testers and lines $\mathrm{x}$ testers turned out to be statistically different for flowering date, plant height, prolificy and yield. Lines 37 and 7 standout with 9.473 and 9.203 t/ha, respectively. Outstanding hybrids showed yields between 9.173 to 9.685 t/ha and included lines 7, 15, 17, 37, 39 and 49. The highest heterosis and best specific combining ability (SCA) were found out in the cross between line $17 \mathrm{x}$ tester 1 with $18,34 \%$ for yield and line 37 was best for general combining ability (GCA) $(0,52)$. Heterosis for flowering date was of $-7,27 \%$ and outstanding for their ACG the lines 14, 15, and 39. For prolificy the line 31 was best than any other line with $52 \%$ of heterosis and ACG too and for ACE the cross of the $17 \mathrm{x}$ tester 1 was superior $(6,28)$.
\end{abstract}

\section{INTRODUCCIÓN}

Las mermas en la producción de maíces tropicales se deben principalmente a factores bióticos y abióticos, entre ellos la alta incidencia de malezas en épocas criticas del cultivo, el ataque de plagas y enfermedades y suelos pobres; otros como la falta de tecnología y las pocas dependencias y/o empresas dedicadas al mejoramiento genético también se hacen partícipe. Dentro de los programas de mejoramiento de germoplasma tropi- cal, se tiene el grave problema de la alta susceptibilidad de las líneas a la depresión endogámica, lo cual provoca la existencia de pocos cultivares con potencial agronómico para el trópico húmedo. Estas son algunas de las razones por el que los estudios en la selección de los nuevos híbridos debe estar basado en la heterosis útil, es decir, en la ganancia genética con base en el material comercial que se este emplee. Varios estudios demuestran que alrededor del $50 \%$ de la ganancia en rendimiento desde los años 30's se ha debido al manejo del

\footnotetext{
1 Financiado por la Universidad Autónoma Agraria Antonio Narro (UAAAN).

2 UAAAN. Buenavista, Saltillo, Coahuila, México. Tel/fax 01(84) 417-73-61. E-mail mmendoza66@hotmail.com.
} 
cultivo (incremento en la dosis de nitrógeno y altas densidades), mientras que el otro $50 \%$ se atribuye al cambio en el genotipo de maíz (Duvick, 1992).

Los híbridos de maíz son citados a menudo como un logro significativo al esfuerzo en los programas de mejoramiento genético. Es así como el término heterosis fue acuñado por Shull desde 1908 y conocido primeramente en maíz; su manifestación puede involucrar un vasto arreglo de caracteres morfológicos, reproductivos, fisiológicos, bioquímicos, tensiones bióticas y abióticas, caracteres de calidad y rendimiento (Vasal y Córdova, 1996). Por otro lado, la magnitud de la heterosis encontrada es de capital importancia para decidir si el mejoramiento es o no práctico y económico de esta manera se iniciará un proceso en la toma de decisiones para el desarrollo de nuevos híbridos. La medición de la heterosis se puede determinar bajo tres formas: 1) a partir del promedio de los progenitores que tiene poco usos para los mejoradores, no así para el genetista cuantitativo que busca el comportamiento de ambos padres $\{\mathrm{H}=(\mathrm{F} 1-\mathrm{PP}) / \mathrm{PP}\}(\mathrm{H}=$ heterosis, $\mathrm{PP}=$ promedio de los padres), 2) en cambio la heterosis basada en el mejor progenitor $\{\mathrm{H}=(\mathrm{F} 1-\mathrm{MP}) / \mathrm{MP}\}(\mathrm{H}=$ heterosis, $\mathrm{MP}=$ mejor progenitor) pero no tiene significado para el genetista, 3) la heterosis útil es considerada la más práctica en el desarrollo de híbridos $\{\mathrm{H}=(\mathrm{F} 1-\mathrm{VC}) / \mathrm{VC}\}$ $(\mathrm{H}=$ heterosis, $\mathrm{VC}=$ material comercial $)$ dado que se requiere comparar los nuevos híbridos con los existentes en el mercado. Por otro lado, Miranda (1997) y Hallauer y Miranda (1988) mencionan que el máximo de heterosis puede ser obtenida en el cruzamiento entre líneas endogámicas completamente contrastantes en sus frecuencias alélicas, es decir, con alelos $\mathrm{P}=0$ en una línea y $\mathrm{P}=1$ en la línea opuesta; este principio también es válido en la cruza entre poblaciones, con la diferencia que ésta es una distribución de frecuencias de alelos en el rango $0=p=1$ para genes que controlan una característica cuantitativa.

Sprague y Tatum (1942) interpretan a la aptitud combinatoria general (ACG) como indicación de los efectos aditivos y a la aptitud combinatoria específica (ACE) de los efectos no aditivos. Desde entonces en la práctica, los términos de aptitud combinatoria han sido utilizados con éxito por el mejorador de plantas; esto ha permitido la elección de aquellos progenitores sobresalientes que participan en el desarrollo de nuevos cultivares.

Bajo estas premisas, en este trabajo de investigación se plantean como objetivos comparar el comportamiento de un grupo de líneas con base en su ACG, determinar cuales son los mejores híbridos triples y cuánta heterosis útil existe en las características bajo estudio.

\section{MATERIALES Y MÉTODOS}

El material genético evaluado fue derivado de dos poblaciones mejoradas, la primera se formó a partir de nueve poblaciones de donde se seleccionaron las mejores progenies de cada una; posteriormente se recombinaron dando lugar al Compuesto Cardel. La segunda población fue formada con variedades representativas de las razas Tuxpeño y Zapalote Chico, a la que se le incorporó el mutante braquítico más tres poblaciones del Istmo de Tehuantepec, originando lo que se conoce como Tutifruti. Después estos dos compuestos se cruzaron (Tutifruti x Compuesto Cardel) derivándose varios cientos de líneas, seleccionando sólo 23 de ellas en forma per-se. Para su evaluación las líneas han sido usadas como hembras con dos probadores, una cruza simple y la variedad Antonio Narro (VAN 555), además se incluyeron tres tratamientos como testigos.

Las características climatológicas y edáficas para la localidad de Villa Ursulo Galván, Ver., son las siguientes: Latitud norte $19^{0} 24^{\prime}$, longitud oeste $97^{0} 24^{\prime}$, altitud de $8 \mathrm{msnm}$, temperatura media de $25,8^{\circ} \mathrm{C}$ y una precipitación de $1017,7 \mathrm{~mm}$; el suelo es tipo feozem y vertisol. La localidad de Carretas, Ver., está situada a una latitud de 19022', una longitud oeste de $96^{035}$ ', a una altura de $129 \mathrm{msnm}$, presenta una temperatura media anual de 26,5 ${ }^{\circ} \mathrm{C}$ y una precipitación media de 979,3 $\mathrm{mm}$; es una área con suelos vertisol y cambisol.

Los tratamientos fueron sembrados en dos repeticiones, siendo la fecha de siembra el día 30 de Junio de 1995 en la localidad de U. Galván, Ver., y para la localidad de Carretas, Ver., el día 13 de Julio del mismo año, se sembraron dos surcos por parcela con una longitud de surco de 4,62 m, una distancia entre surcos de $0,92 \mathrm{~m}$, a una distancia entre plantas de $0,22 \mathrm{~m}$,para un total de 21 plantas por surco. Al momento de la siembra se depositaron dos semillas para después aclarear a una, el área de la parcela experimental fue de 8,13 m, considerando como parcela útil $7,69 \mathrm{~m}$, resultando una densidad de 49.400 plantas/ha. Se realizó un análisis de varianza bloques completos al azar combinado con arreglo factorial, tomando como factor A las líneas y factor B los probadores. La prueba de diferencia mínima significativa (DMS) se aplicó para realizar la comparación de medias. La estimación de la heterosis útil $\{\mathrm{H}=(\mathrm{F} 1-\mathrm{VC}) / \mathrm{VC}\}$ y aptitud combinatoria se realizó de acuerdo a la metodología de Singh y Chaudhary (1977). Se registraron datos para las variables días a flor masculina (días desde la siembra hasta que el $50 \%$ de las plantas de la parcela mostraban anteras dehiscentes), altura de planta (en cm, se midió desde la base de la planta a la hoja bandera antes de la cosecha). La proli- 
ficidad (se estimó en base a una regla de tres, siendo el $100 \%$ el número de plantas cosechadas con respecto al número de mazorcas cosechadas) y el rendimiento se ajustó al 15,5\% de humedad en una muestra de 250 gramos a toneladas por hectárea.

Las labores de preparación del terreno en las dos localidades fueron las acostumbradas en la región: barbecho, rastreo y surcado. La siembra se realizó en forma manual, la dosis de fertilización 120-80-00 fue aplicada manualmente depositando al momento de la siembra el $50 \%$ de nitrógeno y todo el fósforo, en el segundo cultivo de la misma forma fue depositado el resto del nitrógeno. Con respecto, a las labores de cultivo se llevaron a cabo durante todo el ciclo vegetativo, dando prioridad a las primeras etapas de crecimiento y desarrollo, de tal manera que se mantuvo libre de plagas y malezas.

\section{RESULTADOS Y DISCUSIÓN}

De acuerdo con el análisis línea x probador se observó que las líneas, probadores y sus cruzas presentaron diferencias estadísticas $(\mathrm{p}<0,01)$ para floración, altura de planta, prolificidad y rendimiento. Esto refleja la variación que existió dentro del germoplasma bajo evaluación; esta variación permite realizar una buena selección en base a su aptitud combinatoria general (ACG) y aptitud combinatoria específica (ACE), razón por lo cual es importante practicar pruebas tempranas y selección visual (Chávez, 1995). Además, se logra eliminar todas aquellas plantas con características indeseables antes de realizar una mayor inversión (Jenkins, 1935). La heterogeneidad genética se atribuye principalmente al bajo grado de endogamia $\left(\mathrm{S}_{3}\right)$ de las líneas; con respecto a la variación dentro de probadores ésta se debe a la base genética de cada uno (testcross y topcross). Por otra parte, los valores de los cuadrados medios representan los efectos aditivos (ACG) de líneas y probadores y los efectos no aditivos (ACE) están determinados por la interacción línea x probador. Tomando en consideración estos valores, se tiene que los efectos de aptitud combinatoria general (ACG) son más importantes que los no aditivos (ACE) para todas las variables; así también, la varianza de los probadores fue mayor en la variable días a flor y rendimiento; resultados similares reportan Castañon et al. (1997); para la fuente de variación líneas, resultó ser mejor para altura de planta y prolificidad (Cuadro 1).

La respuesta promedio de las líneas mostraron altos rendimientos y al menos 10 de ellas superaron a la media general $(8.479 \mathrm{t} / \mathrm{ha})$ y el $74 \%$ fueron mejores al testigo (8.184t/ha); resultados favorecidos por la característica prolificidad donde el $99 \%$ del germoplasma evaluado fue superior al testigo. En relación a floración existe una diferencia hasta de cuatro días con respecto al mismo; de esta manera, el material genético evaluado no presentó problemas de sincronización floral; en la otra variable altura de planta, las líneas se vieron muy afectadas. En este mismo cuadro se presentan los promedios de las variables bajo estudio en la cruza línea x probador; para rendimiento la cruza simple (probador 1) fue el más productivo reflejándose 0,540 (t/ha) más que en el probador dos (variedad). Resultados similares reportan López (1986), Romero (1996) quienes reportan que el probador de reducida base genética es el más adecuado en la discriminación y clasificación para rendimiento, resultados también afirmados por Cedillo (1985) al utilizar una cruza simple AN2 x AN1 para el trópico seco (Cuadro 2).

Cuadro 1. Análisis de varianza línea x probador en maíz para rendimiento y otras variables en dos localidades del trópico de Veracruz, México, 1995.

\begin{tabular}{|c|c|c|c|c|c|}
\hline F. V. & g. 1. & $\begin{array}{l}\text { Días a flor } \\
\text { masculina }\end{array}$ & $\begin{array}{l}\text { Altura de } \\
\text { planta }\end{array}$ & Prolificidad & $\underset{\text { t/ha }}{\text { Rendimiento }}$ \\
\hline Localidad & 1 & $6,7 *$ & $4863,0 * *$ & $13205,3 * *$ & $55,2 * *$ \\
\hline Rep/loc. & 2 & 0,1 & $1527,0 * *$ & $16,5 * *$ & 0,3 \\
\hline Líneas (A) & 22 & $6,6 * *$ & $816,1 * *$ & $855,2 * *$ & $1,8 * *$ \\
\hline Probador (B) & 1 & $23,0 * *$ & $15,0 * *$ & $577,5 * *$ & $13,4 * *$ \\
\hline Línea x Probador & 22 & $3,4 * *$ & $371,4 * *$ & $332,9 * *$ & $1,9 * *$ \\
\hline Línea x Localidad & 22 & $1,9 *$ & $174,8 * *$ & $236,9 * *$ & 0,7 \\
\hline Probador x Localidad & 1 & 1,2 & $184,0 * *$ & $141,8 * *$ & 1,7 \\
\hline A x B x Localidad & 22 & 0,8 & $286,1 * *$ & $167,5 * *$ & 0,7 \\
\hline Error experimental & 90 & 0,8 & 268,7 & 150,9 & 0,5 \\
\hline Total & 183 & & & & \\
\hline C. V. $(\%)$ & & 1,7 & 7,3 & 10,8 & 8,5 \\
\hline
\end{tabular}

*, ** significativos al 5 y $1 \%$ de probabilidad, respectivamente. 
Cuadro 2. Resultados promedios de las características bajo estudio en la interacción línea x probador en maíz en ciclo Otoño-Invierno, 1995. Veracruz, México, 1995.

\begin{tabular}{|c|c|c|c|c|c|c|c|c|}
\hline \multirow[t]{2}{*}{ Genealogía } & \multicolumn{2}{|c|}{ Días a flor } & \multicolumn{2}{|c|}{ Altura planta } & \multicolumn{2}{|c|}{ Prolificidad } & \multicolumn{2}{|c|}{ Rendimiento } \\
\hline & $\mathbf{P}_{1}$ & $\mathbf{P}_{\mathbf{2}}$ & $\mathbf{P}_{1}$ & $\mathbf{P}_{2}$ & $\mathbf{P}_{1}$ & $\mathbf{P}_{2}$ & $\mathbf{P}_{1}$ & $\mathbf{P}_{2}$ \\
\hline Tutifruti x Comp. Cardel $S_{3}-1$ & 52 & 52 & 238 & 237 & 104 & 107 & 8.973 & 8.274 \\
\hline Tutifruti x Comp. Cardel $\mathrm{S}_{3}-2$ & 52 & 53 & 211 & 213 & 101 & 135 & 7.895 & 8.538 \\
\hline Tutifruti x Comp. Cardel $\mathrm{S}_{3}-3$ & 52 & 52 & 217 & 225 & 112 & 119 & 8.351 & 8.895 \\
\hline Tutifruti x Comp. Cardel $\mathrm{S}_{3}-7$ & 52 & 55 & 239 & 224 & 119 & 107 & 9.509 & 8.898 \\
\hline Tutifruti x Comp. Cardel $\mathrm{S}_{3}-8$ & 52 & 52 & 238 & 221 & 110 & 108 & 8.557 & 8.010 \\
\hline Tutifruti x Comp. Cardel $\mathrm{S}_{3}-10$ & 51 & 53 & 208 & 199 & 101 & 94 & 8.537 & 6.999 \\
\hline Tutifruti x Comp. Cardel $\mathrm{S}_{3}-14$ & 51 & 52 & 215 & 226 & 119 & 118 & 8.388 & 8.227 \\
\hline Tutifruti x Comp. Cardel $\mathrm{S}_{3}-15$ & 51 & 52 & 203 & 208 & 117 & 104 & 9.401 & 7.731 \\
\hline Tutifruti x Comp. Cardel $\mathrm{S}_{3}-16$ & 51 & 51 & 233 & 215 & 110 & 98 & 8.434 & 7.480 \\
\hline Tutifruti x Comp. Cardel $\mathrm{S}_{3}-17$ & 53 & 53 & 243 & 235 & 139 & 110 & 9.685 & 7.667 \\
\hline Tutifruti x Comp. Cardel $\mathrm{S}_{3}-24$ & 54 & 53 & 224 & 236 & 112 & 108 & 8.884 & 8.487 \\
\hline Tutifruti x Comp. Cardel $\mathrm{S}_{3}-27$ & 55 & 54 & 259 & 232 & 109 & 105 & 8.179 & 8.286 \\
\hline Tutifruti x Comp. Cardel $\mathrm{S}_{3}-31$ & 54 & 54 & 230 & 225 & 152 & 136 & 8.640 & 8.061 \\
\hline Tutifruti x Comp. Cardel $\mathrm{S}_{3}-32$ & 51 & 51 & 219 & 233 & 109 & 114 & 8.634 & 8.060 \\
\hline Tutifruti x Comp. Cardel $\mathrm{S}_{3}-37$ & 52 & 54 & 232 & 230 & 134 & 120 & 9.173 & 9.774 \\
\hline Tutifruti x Comp. Cardel $\mathrm{S}_{3}-39$ & 51 & 52 & 224 & 218 & 112 & 113 & 9.223 & 7.981 \\
\hline Tutifruti x Comp. Cardel $\mathrm{S}_{3}-40$ & 51 & 56 & 202 & 229 & 126 & 122 & 7.780 & 7.159 \\
\hline Tutifruti x Comp. Cardel $\mathrm{S}_{3}-45$ & 53 & 54 & 221 & 223 & 104 & 98 & 8.442 & 7.524 \\
\hline Tutifruti x Comp. Cardel $\mathrm{S}_{3}-46$ & 51 & 51 & 229 & 229 & 105 & 105 & 8.642 & 8.113 \\
\hline Tutifruti x Comp. Cardel $\mathrm{S}_{3}-48$ & 52 & 52 & 219 & 242 & 111 & 108 & 8.116 & 8.251 \\
\hline Tutifruti x Comp. Cardel $\mathrm{S}_{3}-49$ & 52 & 54 & 233 & 239 & 120 & 109 & 9.303 & 8.574 \\
\hline Tutifruti x Comp. Cardel $S_{3}-51$ & 53 & 53 & 218 & 236 & 107 & 125 & 8.908 & 8.656 \\
\hline Tutifruti x Comp. Cardel $\mathrm{S}_{3}-52$ & 52 & 52 & 226 & 216 & 135 & 116 & 8.211 & 7.798 \\
\hline Media & 52 & 53 & 225 & 226 & 116 & 112 & 8.690 & 8.150 \\
\hline TH TTC $82^{*}$ & \multicolumn{2}{|c|}{55} & \multicolumn{2}{|c|}{213} & \multicolumn{2}{|c|}{100} & \multicolumn{2}{|c|}{8.184} \\
\hline DMS $(0,01)$ & \multicolumn{2}{|c|}{1,27} & \multicolumn{2}{|c|}{23,01} & \multicolumn{2}{|c|}{17,24} & \multicolumn{2}{|c|}{1,07} \\
\hline
\end{tabular}

Probador 1 (P1=43-46-2-3-2 x V524-223-1-7), Probador 2 (P2= VAN 555), * testigo.

En la estimación de la aptitud combinatoria para rendimiento de acuerdo a la metodología de Singh y Chaudhaury (1977) se encontraron las líneas 37, 7, 49, 45, 52, 17, y 24 como las más sobresalientes en cuanto ACG. En sus cruzas específicas (ACE) con el probador uno sobresalieron la línea 37, 2 y 3; asimismo, la heterosis útil mas alta fue para el material 17 con $18,34 \%$ que equivale a $1.501 \mathrm{t} / \mathrm{ha}$. Existen al menos seis cultivares con los dos probadores que mostraron buen grado de heterosis donde los valores variaron entre 12,08 a 18,34 \%, vigor híbrido bastante aceptable, tomando en cuenta que la estimación se basó en el testigo comercial, ésta se incrementaría sí el calculo fuera en base al mejor progenitor. Para días a floración, la heterosis resultó totalmente negativa con los dos probadores, reflejándose así la selección que se ha realizado para precocidad; en ello sobresalieron el material 46, 14, 15 y 39 por su ACG; también existieron cruzas con el probador dos que presentaron los valores más bajos de ACE (las líneas 7, 10, 37, y 49). Tomando en cuenta la problemática que enfrenta el trópico, la línea 2, $3,10,14,15,16,39,40,45$ y 52 se considera germoplas- ma prometedor para reducir la altura de planta (ACG). La cruza de la línea 40 con el probador uno presentó la mejor ACE (-6,64). En esta misma variable, la heterosis no fue tan favorable considerando que la mayoría de ella fue positiva. Para prolificidad existieron buenas líneas para ACG como la $31(14,96)$ y la $37(6,47)$ y en ACE sobresalieron la 37,31 y 52 con el probador 1 y con el probador dos la línea 2, 8 y 51. En heterosis se obtuvieron valores hasta de $52 \%$ por la línea 31; al respecto Oyervides (1986) concluye que para que el rendimiento se incremente en forma significativa la prolificidad tendrá que ser mayor al $25 \%$, resultados alcanzados por las líneas 17 , $31,34,40,51$ y 52 , por la cual se le considera prometedoras en un programa de hibridación (Cuadro 3). En el Cuadro 4 se muestra como el probador uno (cruza simple) siempre fue superior en heterosis y aptitud combinatoria general al probador dos (variedad) en todas las variables, es decir, fue más productivo. En general, se encontró buen potencial agronómico tomando en cuenta que con el probador uno (cruza simple) se está formando un híbridos triples superiores al testigo. 


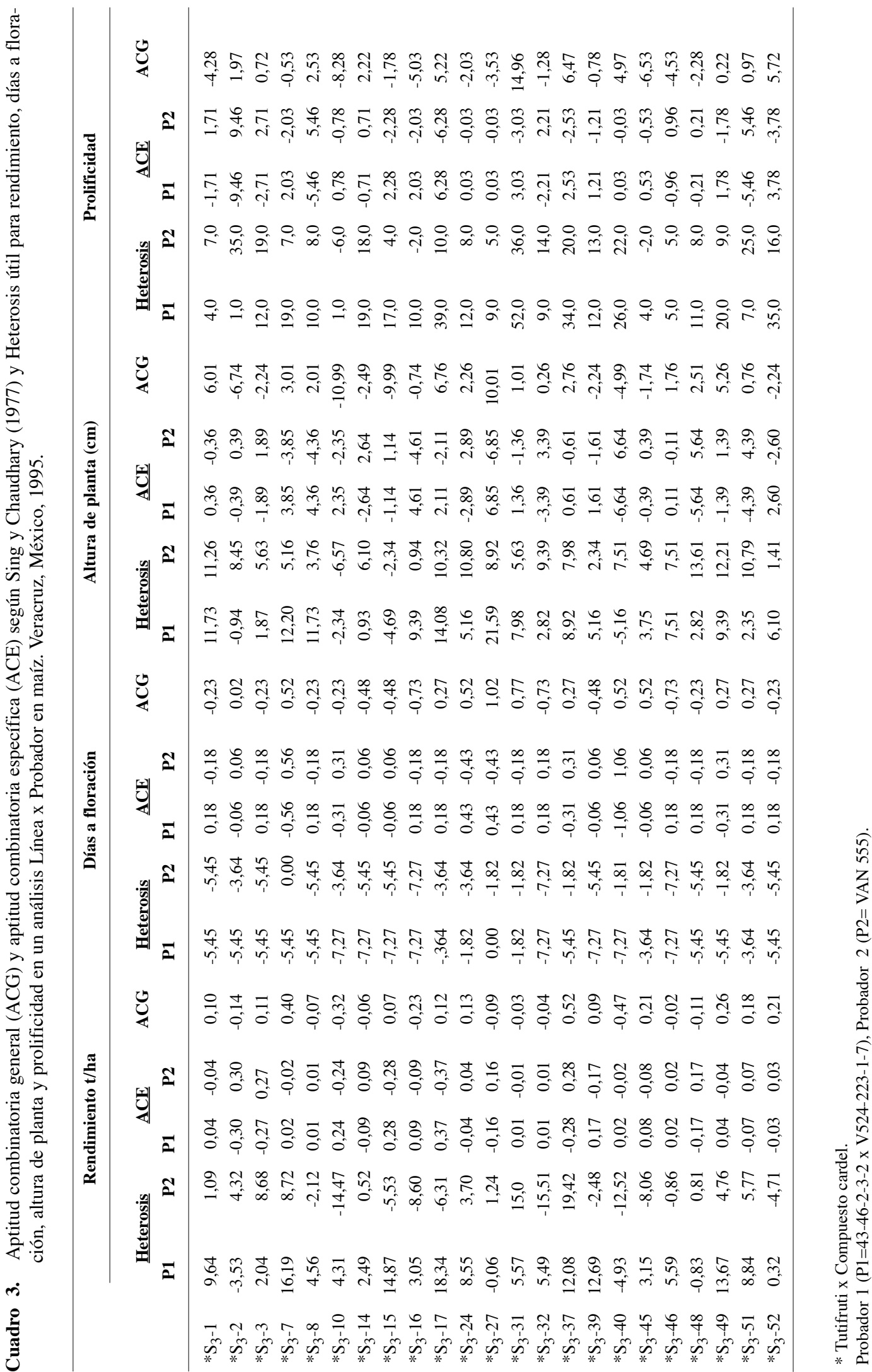


Cuadro 4. Aptitud combinatoria general (ACG) según Sing y Chaudhary (1977) y heterosis útil para probadores en maíz. Veracruz, México, 1995.

\begin{tabular}{lcccccccc}
\hline Características & \multicolumn{2}{c}{ Días a flor } & \multicolumn{2}{c}{ Altura de planta } & \multicolumn{2}{c}{ Prolificidad } & \multicolumn{2}{c}{ Rendimiento } \\
\hline & $\mathrm{P}_{1}$ & $\mathrm{P}_{2}$ & $\mathrm{P}_{1}$ & $\mathrm{P}_{2}$ & $\mathrm{P}_{1}$ & $\mathrm{P}_{2}$ & $\mathrm{P}_{1}$ & $\mathrm{P}_{2}$ \\
Heterosis & $-5,45$ & $-3,63$ & 5,63 & 6,10 & 16,0 & 12,0 & 6,18 & $-0,42$ \\
ACG & $-0,18$ & 0,18 & $-0,11$ & 0,11 & 0,97 & $-0,97$ & 0,13 & 0,13 \\
\hline
\end{tabular}

Probador 1 (P1=43-46-2-3-2 x V524-223-1-7), Probador 2 (P2= VAN 555).

\section{CONCLUSIONES}

Los cruzamientos mostraron gran variabilidad genética, lo que permitió realizar una buena selección; en rendimiento sobresalieron diez líneas, pero las mejores fueron las 37 y 7 con 9.473 y 9.203 t/ha, respectivamente. Los mejores híbridos trilineales presentaron rendimientos que oscilaron entre 9.173 a $9.685 \mathrm{t} / \mathrm{ha}$ y participaron las líneas 7, 15, 17, 37, 39 y 49 con el probador uno (cruza simple).

La heterosis más alta se encontró en la línea 17 x probador uno con 18,34\% para rendimiento; para esta misma variable la mejor ACG $(0,52)$ fue para la línea 37 y el mejor cruzamiento fue entre la línea 17 con el probador 1 (ACE). La heterosis para floración fue de $-7,27 \%$ lo que significa que existe material precoz; otras líneas que sobresalieron por su ACG fueron 14, 15 y 39. La prolificidad fue de gran importancia para que se incrementara el rendimiento, y la línea 31 presentó el valor más alto de heterosis (52\%); este mismo material presentó la mejor ACG, y para ACE la línea 17 x probador uno fue la más interesante $(6,28)$.

\section{LITERATURA CITADA}

CASTAÑÓN, G.; JEFFERS D.; HIDALGO H.; TOSQUY O. H.; ARANO R.; RAYGOZA B. 1997. Line x Tester analysis for drought tolerance in tropical maize $I n$ : Book of Abstracts. The genetics and exploitation of heterosis in crops. An international symposium. Mexico, D. F., Mexico. pp. 46-47.

CEDILLO, G. V. 1985. Comportamiento de 26 líneas de maíz derivadas de V-524 en un estudio de aptitud combinatoria con tres tipos de probadores. Tesis Lic. UAAAN. Saltillo, Coahuila, México. 73 p.

CHÁVEZ A, J .L. 1995. Mejoramiento de Plantas II. 2a. ed. Editorial Trillas. México, D. F:
DUVICK, D. N. 1992. Genetic contributions to advances in yield of U. S. maize. Maydica 37:69-79.

HALLAUER, A. R.; J. B. MIRANDA. 1988. Quantitative genetics in maize breeding. Iowa Sate University. Press/Ames. pp. 159-294.

JENKINS, M. T. 1935. The effect of inbreeding and selection within inbred lines of maize upon the hybrids made after successive generations of selfing. Iowa State J. Sci. 3:429-450.

LÓPEZ, P. E. 1986. Comparación entre diferentes probadores para evaluar líneas de maíz. Folleto de Divulgación. Vol. 1, No. 7. UAAAN. Saltillo, Coahuila, México. pp. 2-5.

MIRANDA F. J. B. 1997. Inbreeding and heterosis. In: Book of Abstracts. The genetics and explotation of heterosis in crops. An international symposium. Mexico, D. F., Mexico. pp. 12-13.

OYERVIDES, G. A. 1986. Estudio de la importancia económica de tres caracteres morfológicos del maíz. Tesis de Maestría. Colegio de Postgraduados. Chapingo, México. 151 p.

ROMERO, C. M. G. 1996. Evaluación de líneas tropicales de maíz en forma perse y en cruza con dos probadores para determinar su aptitud combinatoria. Tesis Profesional. UAAAN. Saltillo, Coahuila, México. 74 p.

SINGH, R. K.; B. D. CHAUDHARY. 1977. Biometrical methods in quantitative genetic analysis. Kalyani Publishers. Revised Edition. 1979. Printed in India. pp. 191-200.

SPRAGUE, G. F.; L. A. TATUM. 1942. General versus specific combining ability in single crosses of corn. J. Am. Soc. Agron. 34:923-932.

VASAL, S. K.; H. CÓRDOBA. 1996. Heterosis en maíz: Acelerando la tecnología de híbridos de dos progenitores para el mundo en desarrollo. Memoria del Curso Internacional de Actualización en Fitomejoramiento y Agricultura Sustentable. 1a. Edición. UAAAN. Buenavista, Saltillo, México. pp. 32-55. 